\title{
Correction to: A high-cholesterol diet promotes steatohepatitis and liver tumorigenesis in HCV core gene transgenic mice
}

\author{
Xiaojing Wang ${ }^{1,2} \cdot$ Naoki Tanaka $^{1,3}$ (1) $\cdot$ Xiao Hu $^{1,4} \cdot$ Takefumi Kimura $^{5} \cdot \mathrm{Yu} \mathrm{Lu}^{1} \cdot$ Fangping Jia $^{1} \cdot$ Yoshiko Sato $^{6}$. \\ Jun Nakayama ${ }^{6} \cdot$ Kyoji Moriya $^{7} \cdot$ Kazuhiko Koike $^{8} \cdot$ Toshifumi Aoyama $^{1}$
}

Published online: 8 June 2019

○) Springer-Verlag GmbH Germany, part of Springer Nature 2019

\section{Correction to: Archives of Toxicology}

https://doi.org/10.1007/s00204-019-02440-7

In the original publication of the article, there is a mistake in Fig. 2e and the author would like to correct it. It has been corrected and replaced in the HTML and PDF versions of the manuscript.

The corrected Fig. 2 is given below.

Publisher's Note Springer Nature remains neutral with regard to jurisdictional claims in published maps and institutional affiliations

The original article can be found online at https://doi.org/10.1007/ s00204-019-02440-7.

Naoki Tanaka

naopi@shinshu-u.ac.jp

1 Department of Metabolic Regulation, Shinshu University School of Medicine, Matsumoto 390-8621, Japan

2 Department of Gastroenterology, Lishui Hospital, Zhejiang University School of Medicine, Lishui, Zhejiang, People's Republic of China

3 Research Center for Social Systems, Shinshu University, Matsumoto, Japan

4 Department of Pathophysiology, Hebei Medical University, Shijiazhuang, People's Republic of China

5 Department of Gastroenterology, Shinshu University School of Medicine, Matsumoto, Japan

6 Department of Molecular Pathology, Shinshu University School of Medicine, Matsumoto, Japan

7 Department of Infection Control and Prevention, The University of Tokyo, Tokyo, Japan

8 Department of Gastroenterology, The University of Tokyo, Tokyo, Japan 

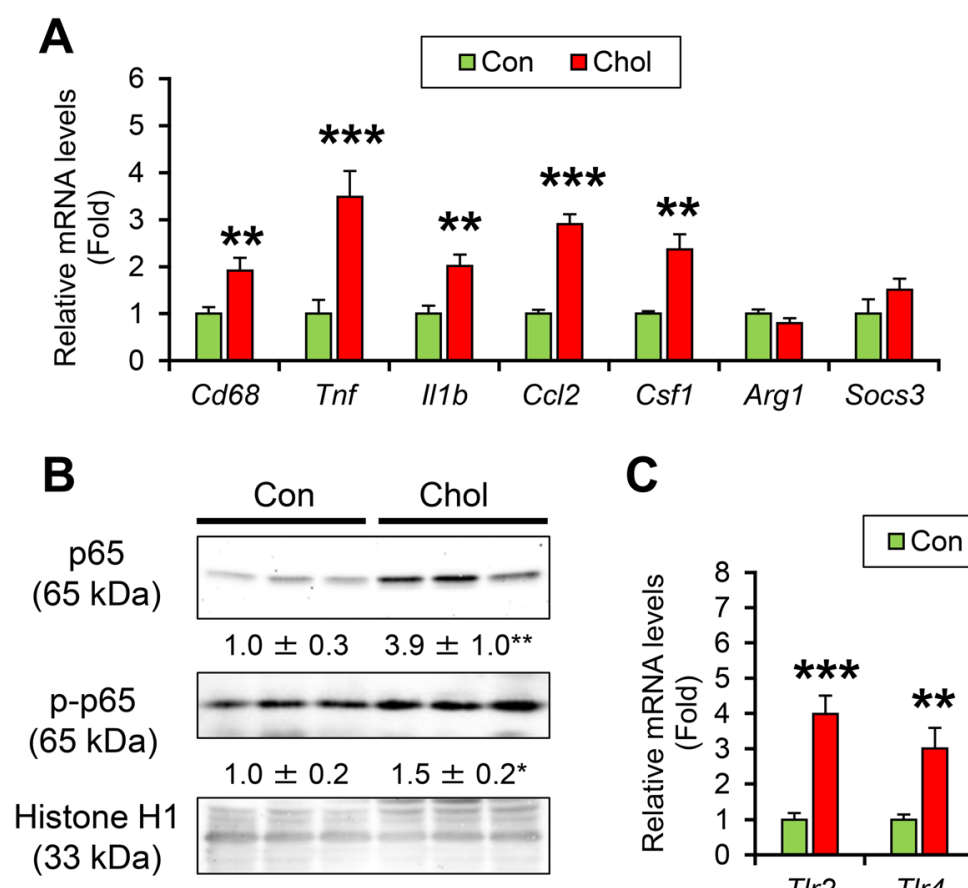

C
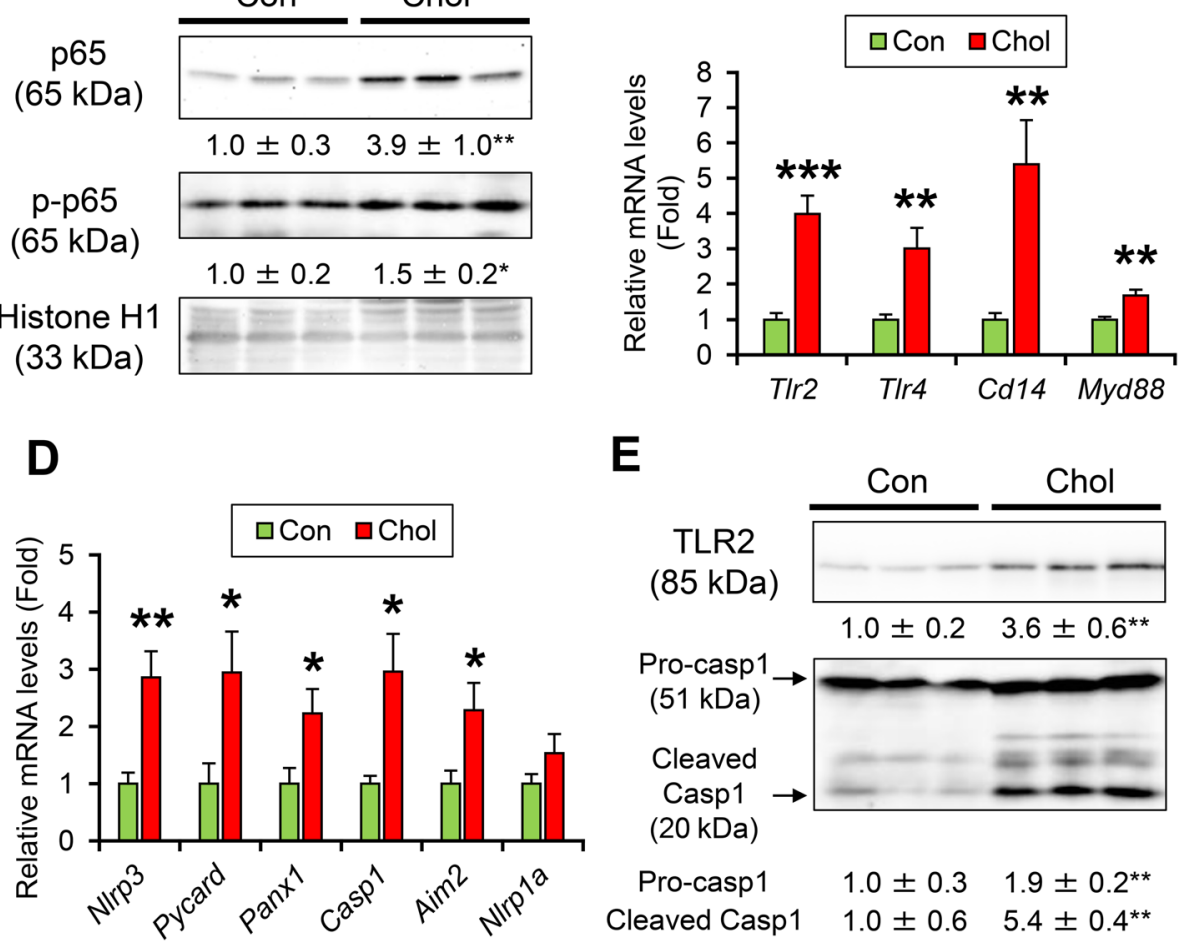

$\mathbf{E}$

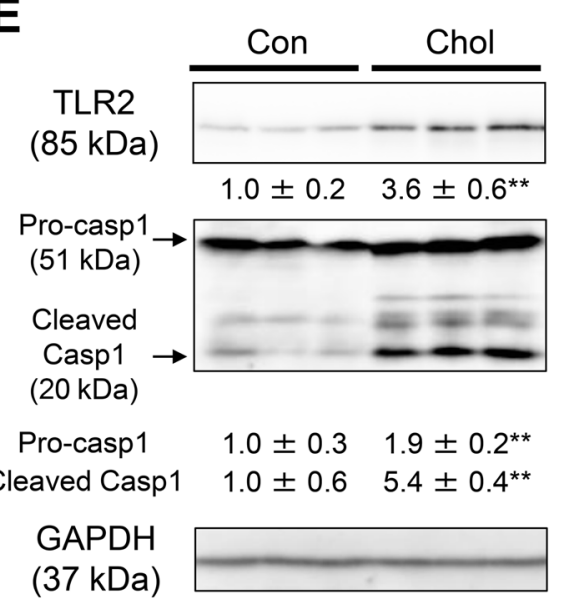

Fig. 2 Aggravated hepatic inflammation in the liver of HCVcpTg mice after a 15-month high-cholesterol diet. a qPCR analysis of genes associated with inflammation. b Immunoblot analysis of NF- $\mathrm{KB}$ p65 and phosphorylated p65 (p-p65). Liver nuclear fractions $(20 \mu \mathrm{g}$ of protein) were loaded into each well. The band of histone H1 was used as a loading control. Band intensities were measured densitometrically, normalized to those of the loading controls, and subsequently expressed as values relative to those of control diet mice. Results were obtained from two independent immunoblot experiments. c qPCR analysis of genes associated with TLRs. d qPCR analysis of genes associated with inflammasomes. e Immunoblot analysis of TLR2 and caspase 1 . Whole liver homogenates ( $45 \mu \mathrm{g}$ of protein) were loaded into each well. The caspase 1 antibody could detect procaspase 1 and cleaved (activated) caspase 1. The band of GAPDH was used as a loading control. Band intensities were measured densitometrically, normalized to those of the loading controls, and subsequently expressed as values relative to those of control diet mice. Results were obtained from two independent immunoblot experiments. All mRNA levels were normalized to $18 \mathrm{~S}$ ribosomal RNA levels and subsequently expressed as values relative to those of control diet mice. Values are expressed as the mean \pm SEM. $* P<0.05$, $* * P<0.01, * * * P<0.001$ between control diet and high-cholesterol diet HCVcpTg mice 